\title{
Detection of Babesia divergens in southern Norway by using an immunofluorescence antibody test in cow sera
}

\author{
Gunnar Hasle ${ }^{1,2^{*}}$, Gunnar A Bjune ${ }^{2}$, Dan Christensson ${ }^{3}$, Knut H Røed ${ }^{4}$, Anne C Whist ${ }^{5}$, Hans P Leinaas ${ }^{1}$
}

\begin{abstract}
Background: The incidence of bovine babesiosis, caused by Babesia divergens (Apicomplexa: Piroplasmida) has decreased markedly since the $1930 \mathrm{~s}$, but may re-emerge as a consequence of climate change and changes in legislation and pasturing practices. This is a potentially serious disease, with both economical and animal welfare consequences. Therefore, there is a need to survey the distribution of $B$. divergens.

Methods: We tested sera from 306 healthy pastured cows from 24 farms along the southern Norwegian coast by using an indirect immunofluorescence lgG antibody test (IFAT). Fractions of seropositive cows were compared by calculating $95 \% \mathrm{Cl}$.

Results: The results of this test showed that $27 \%$ of the sera were positive for $B$. divergens antibodies. The fraction of antibody-positive sera that we detected showed a two-humped distribution, with a high fraction of positives being found in municipalities in the western and eastern parts of the study area, while the municipalities between these areas had few or no positive serum samples.

Conclusions: Neither the farmers' observations nor the Norwegian Dairy Herd Recording System give an adequate picture of the distribution of bovine babesiosis. Serological testing of cows by using IFAT is a convenient way of screening for the presence of $B$. divergens in an area.
\end{abstract}

\section{Background}

Though the incidence of bovine babesiosis is low in Norway, these pathogens have immense economic importance throughout the world, with the highest prevalence being found in the tropics [1]. The costs associated with this infection are associated with mortality, ill-thrift, abortions, loss of milk and meat production as well as with measures taken to control its spread [2]. Babesia divergens is the main cause of bovine babesiosis in northern Europe [3], although B. major, occurs in southeast England, Holland and the Friesian Islands in Germany [4]. Babesia species are intraerythrocytic protozoa that cause fever, haemoglobinuria (redwater) and anaemia in cattle that are exposed to the parasite as adults. Calves are relatively resistant to $B$. divergens $[5,6]$ and exhibit mild or no effects of the disease, while

\footnotetext{
* Correspondence: hasle@reiseklinikken.com

'Department of Biology, University of Oslo, P.O. Box 1050 Blindern, N-0316

Oslo, Norway

Full list of author information is available at the end of the article
}

infected adults may have a high mortality $[7,8]$. Babesia spp. can cause serious infections in humans who do not have a functioning spleen or who are immunocompromised as a result of immunosuppressive drugs, malignancy or HIV-infection [9]. The only case of human $B$. divergens diagnosed in Norway is a splenectomised veterinarian in Western Norway in 2007 (personal communication, Kristine Mørch, Haukeland University Hospital).

Cattle are the only natural vertebrate host for $B$. divergens. Reindeer and gerbils, and splenectomised individuals of other species may be infected experimentally. Sheep, wild cervids and rodents that occur in the area where it is distributed are all considered to be resistant to $B$. divergens [3]. However, this issue is controversial, as new studies indicate that roe deer and red deer may be infected by $B$. divergens $[10,11]$. The vector of $B$. divergens in Western Europe is Ixodes ricinus (Acari: Ixodidae) [3], which can parasitise a wide range of vertebrates [12]. Vertebrate hosts may act as vehicles for 
spreading Babesia-infected ticks, though only adult females of $I$. ricinus can become infected with $B$. divergens from cattle [13]. Transovarial and transstadial transmission of $B$. divergens occur in I. ricinus [14], and the infection can last for at least two generations [13]. Thus, these ticks may also represent a reservoir of the parasites, though only a small percentage of the larvae from the infected females usually carry the pathogen [13]. Each female of I. ricinus produces approximately 2,000 eggs [15], so there will be a correspondingly high mortality from one stage to the next in a stable tick population. Supposing a maximum 3 years generation time of $I$. ricinus and a maximum of three generations of parasite survival through transovarial transmission, the pathogen would, therefore, be expected to gradually disappear within a decade in areas where there are no vertebrate hosts present to transmit the infection to the ticks. After recovering from acute babesiosis, cattle may sustain a low level of parasitaemia for at least two years, which may be followed by the development of immunity to the parasite, without any detectable parasites in the blood [16]. Opsonising antibodies play an important role in protecting hosts against $B$. divergens infection, but the acquired immunity is not dependent on circulating antibodies, and in vitro tests have demonstrated a role of T-lymphocytes in protection against the disease. Antibody levels generally fall below the level of detection within six months after treatment [2]. The longlasting host-parasite interaction results in the cattle acting as an effective reservoir of the parasites [17].

In Norway, the law does not mandate obligatory notification of bovine babesiosis, and no systematic study on the distribution of this parasite has been undertaken since the work of Thambs-Lyche from 1933-1940 where 1388 cases per year were reported [18]. One way of estimating the number of cases of this infection that exist today is by looking at sales of imidocarb, a veterinary medicine used to treat bovine babesiosis. Approximately 300 vials of $1200 \mathrm{mg}$ imidocarb are sold per year in Norway (Bjørn Loe, Schering-Plough, personal communication), and this amount would be sufficient for treatment of a maximum of 600 individuals. Alternatively, data recorded at the Norwegian Dairy Herd Recording System (NDHRS) can be examined, since every cow in Norway is assigned an individual Cow Health Card on which all diseases are recorded by veterinarians or farmers and then reported to the NDHRS. This system has been in operation nationally since 1975 [19], and the health code and date of all disease treatment events are maintained in a central database. From 1996-2008, 121 cases of bovine babesiosis were reported in the NDHRS per year. Thus, both of these estimation methods indicate that the incidence of bovine babesiosis in Norway has fallen markedly since the $1930 \mathrm{~s}$. This decrease coincides with, and may be explained by, a marked decrease in pasturing of cattle. In 1938, almost all of the 1.3 million cattle population in Norway were pastured regularly, whereas only 220,000 of the present 920,000 cattle population are pastured during the summer $[20,21]$. A decrease in bovine babesiosis has also been documented in Ireland. Gray et al. suggested that this might be due to a combination of several factors, such as an increase in average farm size and destruction of ticks' habitat by increased sheep pasturing. On the other hand, they suggested that the rate of clinical disease is low in western Ireland because of enzootic stability, i.e., the herds are naturally immune [22].

Bovine babesiosis is regarded as a limited problem in Norway, being confined to coastal areas north to southern Nordland county [23]. However, there may be a locally elevated risk of contracting babesiosis, which might be an argument against importing adult cows from inland localities where redwater does not occur and that, therefore, will not harbour any acquired immunity to the disease. In addition, changes in climate and pasturing practices could also lead to an increase in the incidence and distribution of bovine babesiosis. As the distributional range of ticks in Scandinavia expands [24], bovine babesiosis may be introduced into areas where livestock do not have a natural immunity to infection. We have no sound scientific data in support of an expansion of tick distribution in Norway, although this has been documented in Sweden [24]. Moreover, since 2004 all tie-stalled cattle in Norway have been required to be pastured for a minimum of 8 weeks during the summer [25], and this same legislation will also apply to cows in free-stalls by 2013, which could lead to an increase in bovine babesiosis. Because of these changes an updated map of the distribution of this parasite is needed for the purpose of better management. The distribution of $B$. divergens could be mapped by testing for the presence of the pathogen in ticks using PCR. Lundsett [26] tested 439 flagged ticks along the southern Norwegian coast and found only one tick that was positive for $B$. divergens using this method. Radzijevskaja [27] found no B. divergens in 91 ticks (16 adults, 75 nymphs) collected in Jomfruland, where we found that redwater is perceived to be a problem by farmers. Thus, testing ticks for $B$. divergens directly is both laborious and costly, and would require analysis of a very large number of ticks.

The aim of this study was to use a well-established indirect immunofluorescent antibody test (IFAT) to detect the presence of $B$. divergens antibodies in blood sera [28], and to evaluate this method as a means of mapping the distribution of the pathogen by comparing our results with information obtained either through reporting through the NDHRS or by interviewing the farmers. 


\section{Materials and methods}

The study area consisted of farms with cows that were pastured in wooded areas within the previously established distribution of bovine babesiosis [29]. Twentyfour farms scattered along most of the southern Norwegian coast west of the Oslo Fjord (Figure 1) were included in the study. Farms using hillside or wooded areas for pasturing were identified with the help of local agricultural authorities. None of the farmers who were asked to participate in the study refused. All the farmers confirmed that ticks occur on their farms, and the cattle were pastured on the property. All of the 306 cows included in the study were healthy and at least one year old when tested. On one farm (Farm 23), all the cows had been purchased one year prior to the study from various inland localities and had been pastured for just one season at this farm. I. ricinus is distributed mainly near the coast in this part of Norway. The study

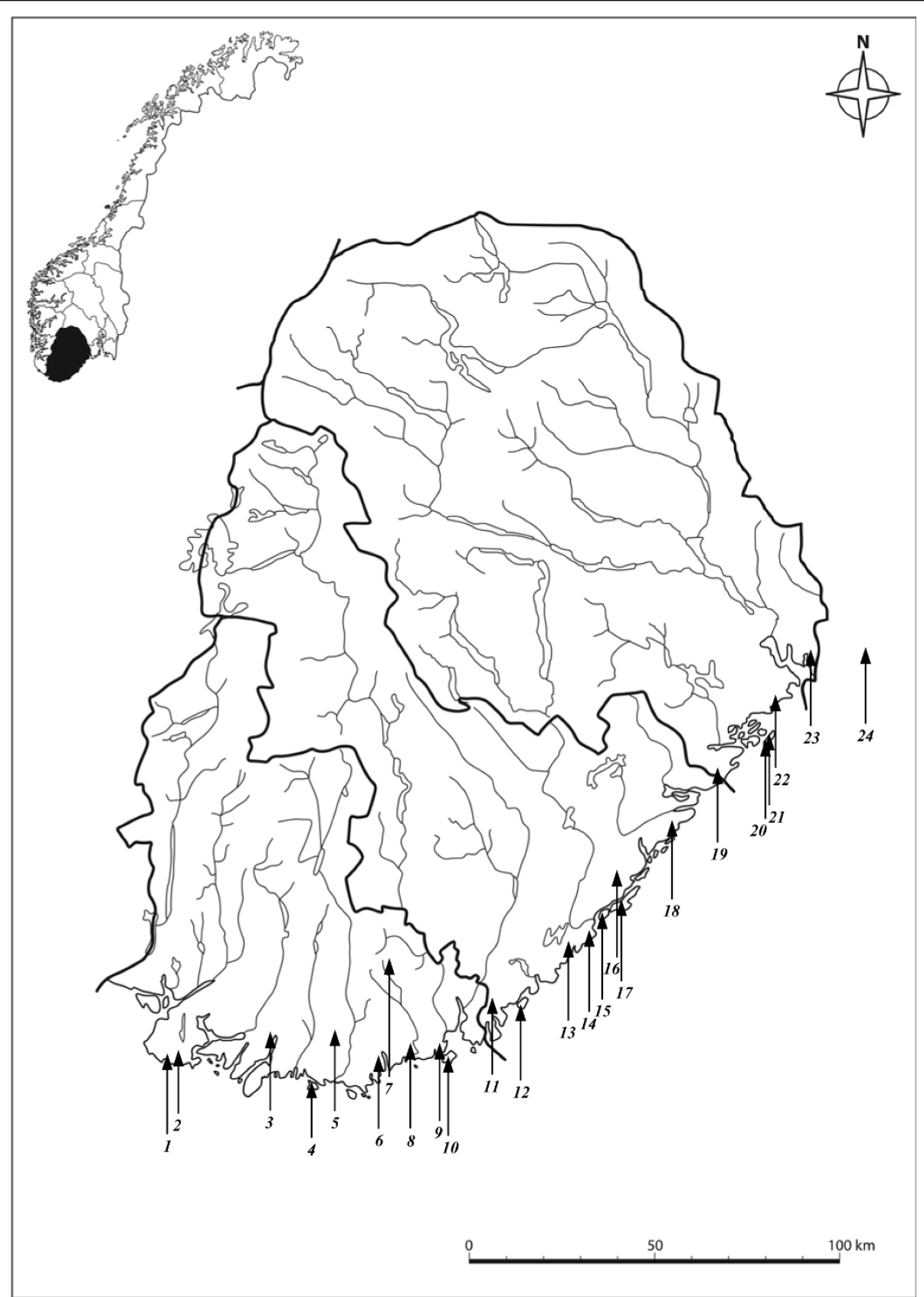

Figure 1 Map of Vest-Agder, Aust-Agder and Telemark counties, with study localities numbered from west to east (Table 1). Farm number 24 is in Vestfold County. (Copyright, map basis: Cappelen Damm as.). 
included one inland farm approximately 30 kilometres from the sea (Farm 7) that was included because human Lyme borreliosis had been reported in this municipality, thus indicating the presence of ticks, according to the Norwegian Surveillance System for Communicable Diseases (MSIS) [30]. Blood samples were collected in May 2004 on farms 20 and 21, and samples were collected from all other sites in October and November 2005. The blood samples were stored at $4{ }^{\circ} \mathrm{C}$ within a few hours after collection, and the serum portion of the samples was separated and frozen within 72 hours.

All of the sera were tested using an indirect immunofluorescent antibody test (IFAT) [28] for IgG as described by Christensson [31,32], and Christensson and Moren [33] with the following modifications: Antigen was prepared in 2002 from blood of a calf infected with Babesia divergens with approx. $10 \%$ infected erythrocytes as described by Christensson [32]. The antiserum used was was FITC conjugated rabbit anti bovine IgG, produced by ICN Cappel, code 55280, lot 03683, diluted at $1 / 200$ to give comparable readings with control sera used by Christensson and Morén [33]. Control sera were obtained from calves used for vaccine production in the year 2001 drawn before infection and four weeks after having showed acute parsitaemia. Negative control serum showed no or uncertain reaction at a dilution of $1 / 20$ or higher. The positive control sera had an endpoint titre of $1 / 1280-1 / 2560$. For each day of reading IFAT-slides a negative control at $1 / 40$ and a positive control at 1/40,1/160 and 1/1280 were included. As the purpose of the test was to identify seropositive/seronegative animals sera were read at dilutions at $1 / 40$ and $1 /$ 160. Slides were read blindly and scored by Christensson as having uncertain $(+)$, positive $(++)$ or strongly positive immunofluorescence $(+++)$, at dilutions of 1:40 and 1:160. To minimise the risk of false positives, only sera with a minimum +++ score at a dilution of 1:40 were counted as positive.

Farmers were interviewed to determine if there had been cases of redwater on their farms and if they had experienced redwater in cows that were imported to the farm. Data on the cases of babesiosis in the included farms were obtained from the NDHRS.

To test the suitability of using PCR on full blood, we chose samples for a pilot study from four farms where redwater was common, according to the local farmers, and DNA from $100 \mu \mathrm{l}$ from 20 samples of frozen EDTA-blood, and 25 samples of $100 \mu \mathrm{l}$ blood clot, frozen after spinning and removal of the serum, were isolated in a spin-column, using DNeasy Blood \& Tissue Kit (Qiagen), and eluated to $200 \mu \mathrm{l}$, according to the manufacturer's protocol. The isolation of DNA contained a lysis step and washing. Five $\mu \mathrm{l}$ of the eluate was run in $B$. divergens real-time PCR for 40 cycles with primers BdiF, BdiR and BdiT. The PCR was performed by Telelab (Skien, Norway), using an in-house method, as described by Lundsett [26]. The laboratory used a synthetic amplicon with the sequence of $B$. divergens, serially diluted in human DNA as a positive control. The reaction mix and human DNA was used as a negative control. The observed cutoff for detection was 30 $B$. divergens DNA copies, i.e. 15 to 30 individual cells, depending of whether they are asexual, diploid cells or sexual, haploid cells.

Exact confidence intervals for binomial proportions were calculated using the principles introduced by Clopper and Pearson [34] and implemented in R (R Development Core Team, 2008).

\section{Results}

Of the 306 sera that we tested, 84 (27\%) had positive IFAT results. A high percentage of these positive results were found in the western and eastern ranges of the study area, and a much lower rate of positive test results was found in the middle range of the study area (Table 1; Figure 2). Farm 23 had 3 positive test results among

Table 1 Municipality of the test localities in Figure 1 and test results of indirect immunofluorescence antibody tests (IFAT) for Babesia divergens.

\begin{tabular}{|c|c|c|c|c|c|}
\hline Farm & Municipality & $\mathrm{Neg}$ & $\operatorname{Pos}^{1}$ & $\mathbf{N}$ & $\%$ pos \\
\hline 1 & Farsund & 6 & 5 & 11 & 45 \\
\hline 2 & Farsund & 6 & 9 & 15 & 60 \\
\hline 3 & Lyngdal & 6 & 2 & 8 & 25 \\
\hline 4 & Mandal & 0 & 9 & 9 & 100 \\
\hline 5 & Mandal & 5 & 7 & 12 & 58 \\
\hline 6 & Søgne & 2 & 7 & 9 & 78 \\
\hline 7 & Songdalen & 13 & 1 & 14 & 7 \\
\hline 8 & Søgne & 3 & 1 & 4 & 25 \\
\hline 9 & Kristiansand & 9 & 0 & 9 & 0 \\
\hline 10 & Kristiansand & 3 & 0 & 3 & 0 \\
\hline 11 & Lillesand & 16 & 2 & 18 & 11 \\
\hline 12 & Lillesand & 8 & 0 & 8 & 0 \\
\hline 13 & Grimstad & 29 & 2 & 31 & 6 \\
\hline 14 & Grimstad & 19 & 0 & 19 & 0 \\
\hline 15 & Arendal & 10 & 0 & 10 & 0 \\
\hline 16 & Arendal & 14 & 1 & 15 & 7 \\
\hline 17 & Arendal & 6 & 4 & 10 & 40 \\
\hline 18 & Risør & 4 & 13 & 17 & 76 \\
\hline 19 & Kragerø & 11 & 1 & 12 & 8 \\
\hline 20 & Kragerø & 6 & 12 & 18 & 67 \\
\hline 21 & Kragerø & 2 & 5 & 7 & 71 \\
\hline 22 & Bamble & 12 & 0 & 12 & 0 \\
\hline 23 & Porsgrunn & 13 & 3 & 16 & 19 \\
\hline 24 & Larvik & 19 & 0 & 19 & 0 \\
\hline Total & & 222 & 84 & 306 & 27 \\
\hline
\end{tabular}

1. IFAT IgG titres scored as 1:40 (+++) or higher are defined as positive. 


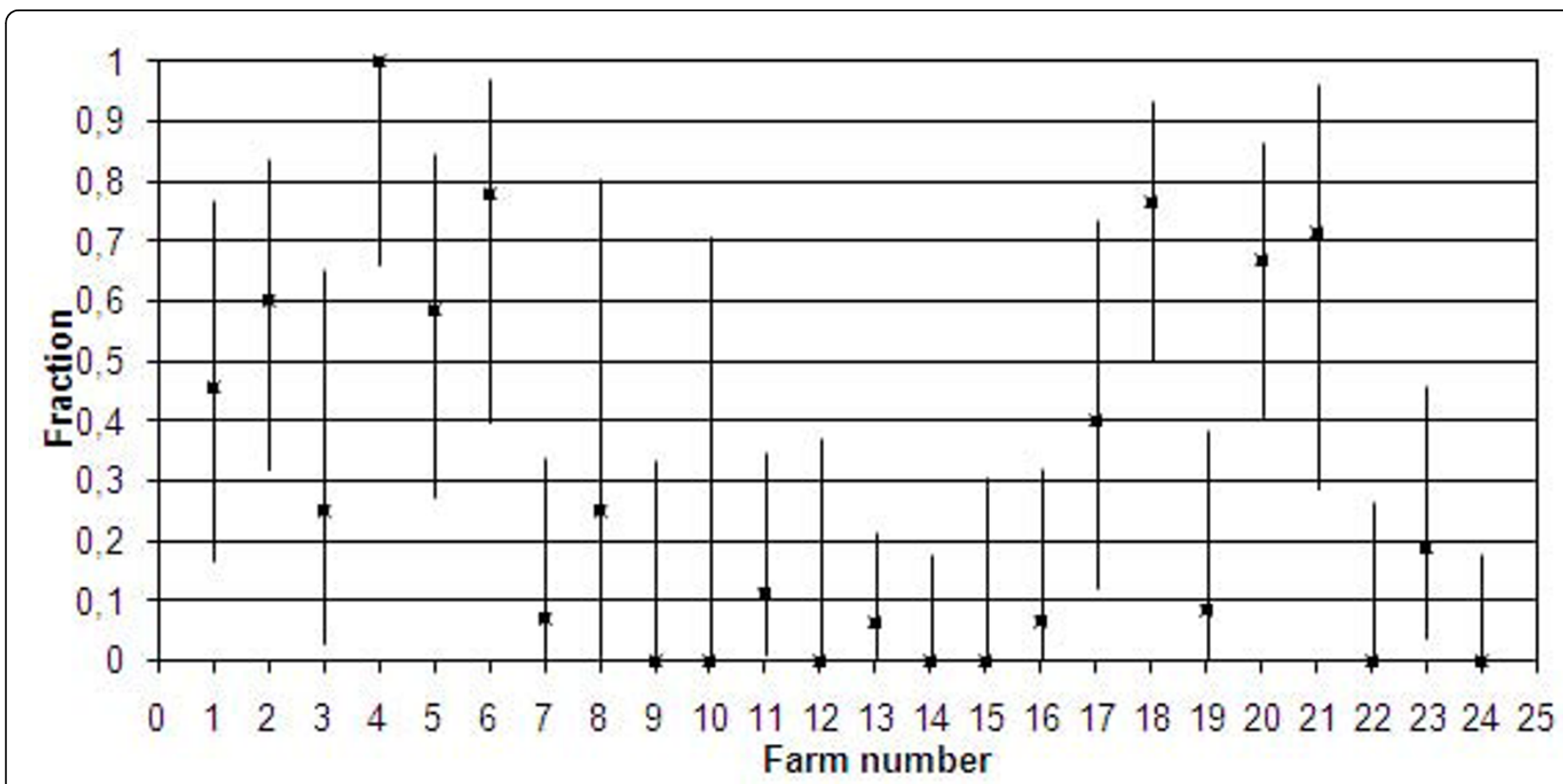

Figure 2 Fraction of cows positive for Babesia divergens IFAT IgG antibodies at a titre of 1:40 (+++) or higher in 24 different farms along the southern Norwegian coast, arranged form west to east. Error bars: $95 \%$ confidence intervals.

the 16 cows that had been imported from inland localities one year before the study, indicating that there is a substantial risk of babesiosis in their present locality. The presence of $B$. divergens was confirmed by IFAT in a total of 17 of the 24 farms we tested. Farmers had observed redwater in only ten of the farms where $B$. divergens was detected, and only four of these cases of redwater had been recorded by the NDHRS (Figure 3). All of the cows on one of the farms in the study were $B$. divergens-antibody positive, though the owner had never seen any cases of redwater. We detected $B$. divergens antibodies in 17 of the 25 cows that we tested on Jomfruland, where Radzijevskaja [27] found no infected ticks.

The PCR pilot study gave no positive results.

\section{Discussion}

In Norway and Sweden the only cattle Babesia reported is $B$. divergens $[35,36]$. With regard to this and the strong reaction to the antigen used we assume that the seropositive animals were/had been infected with the species Babesia divergens. Our results demonstrate that testing of cattle for seropositivity to $B$. divergens is a far better method for mapping the distribution of this pathogen than using indirect methods, such as interviewing farmers or relying on the NDHRS. When it presents clinically, redwater is easily recognizable by farmers and veterinarians, and because prompt treatment is usually required to prevent deleterious effects of the disease, veterinarians often treat the disease without performing any laboratory tests. There are few data available on the attack rate of bovine $B$. divergens infections. Our data indicate that there are many subclinical cases of $B$. divergens infection, which is in agreement with previous studies on outbreaks $[7,37]$ and in stable infected herds [38]. An extensive study of $B$. divergens seroprevalence was conducted in Northern Ireland, showing an overall seroprevalence of $31,8 \%$ [39], i.e., close to the overall seroprevalence in our limited material. A second study carried out in Northern Ireland [40] found consistent estimates when comparing results from a farm survey, a veterinary practise survey and seroprevalence data, with an estimated clinical incidence of $0,26 \%$ per year. The number of cases in the Agder counties, according to the NHDRS, is 18.4 cases per year in a population of ca. 10400 dairy cows (Statistics Norway, http://www.ssb.no/emner/10/04/10/jt1999/tab-2001-0403-07.html, Jordbrukstelling 1999), which would give an incidence of $0.18 \%$ per year. Our results indicate an incomplete registration of cases of redwater in the NHDRS, possibly because veterinarians are not always consulted e. g. during the dry period, in mild cases of redwater, or that the farmers fail to observe redwater while the cows are out at pasture. The farms that we included in our study were not randomly selected, but were chosen because the pastures were in wooded areas, and were situated near the coast in the distribution area of $I$. ricinus in Norway. They would therefore be expected to have more babesiosis than average farms in the same counties. 


\begin{tabular}{|r|l|l|l|}
\hline Farm & $\begin{array}{l}\text { IFAT IgG } \\
\text { positive }\end{array}$ & Noticed & Notified \\
\hline 1 & & & \\
\hline 2 & & & \\
\hline 3 & & & \\
\hline 5 & & & \\
\hline 6 & & & \\
\hline 7 & & & \\
\hline 8 & & & \\
\hline 9 & & & \\
\hline 10 & & & \\
\hline 11 & & & \\
\hline 12 & & & \\
\hline 13 & & & \\
\hline 14 & & & \\
\hline 15 & & & \\
\hline 16 & & & \\
\hline 17 & & & \\
\hline 18 & & & \\
\hline 19 & & & \\
\hline 20 & & & \\
\hline 21 & & & \\
\hline 22 & & & \\
\hline 23 & & & \\
\hline 24 & & & \\
\hline
\end{tabular}

Figure 3 Comparison of three sources of information for the occurrence of babesiosis on the farms in this study. IFAT IgG positive: At least one cow positive for IFAT Babesia divergens IgG. Noticed: Farmers' statement that redwater occurs in cows on the farm or is detected when adult cattle are imported to the farm. Notified: Clinical cases registered on the diary cow health cards, compiled by the Norwegian Dairy Herd Recording System from 1996-2008.

Because cows are parasitised by hundreds of ticks in the course of a season, and a single bite from an infected tick is sufficient for transmission of Babesia, [41] cows are likely to contract $B$. divergens if it is present in their pasturing areas. The screening of cows for $B$. divergens infection would therefore be expected to be a sensitive method for detecting the presence of the parasite in a locality, if testing is performed at a time of the year when Babesia-antibodies are at the highest. Serum samples that we collected on Jomfruland in May were not directly comparable to those that we collected in October and November, as the May samples could either contain persistent antibodies from the previous year, or there might be early infections from the same year. The mean temperature April $1^{\text {st }}-15^{\text {th }}$ was $5.3^{\circ} \mathrm{C}$, and no temperatures of below $0^{\circ} \mathrm{C}$ were recorded (The Norwegian Meteorological Institute), which means that tick questing may well have occurred during this period. With an incubation time of 1-3 weeks [3], seroconversions during May 2004 would be expected to occur. As we tested only once for each locality we did not demonstrate the seasonal and yearly variation of antibodies described by l'Hostis et al. [38]. Further studies are needed to decide which month would be optimal for detecting the presence of $B$. divergens in a locality along the Southern Norwegian coast. However, ticks are still parasitizing the cows in October and November and these months would therefore be expected to be a good choice for detecting $B$. divergens antibodies.

The sensitivity of serologic testing for detecting $B$. divergens will depend on the cut-off level that is set for a positive score on the test. At a cut-off level of 1:40 (+ + ) the sensitivity and specificity of an individual antibody test are reported to be $100 \%$ and $97 \%$, respectively [32]. Setting the cut-off value at this level would, therefore, likely result in the detection of a few false positives due to non-specific cross reactivity. This problem is illustrated by our results on Farm 24, where only one cow was found to be positive at the detection level of 1:40 $(++)$, and there were no positive tests at more stringent detection levels. This result could represent either a false positive or a low titre in a cow that was infected a long time ago. Because the aim of this study was to be able to detect the present occurrence of $B$. divergens at a particular locality, a high sensitivity for detecting the pathogen on a given farm is desirable, and the number of cows tested is crucial. By testing a median of twelve cows per locality, we were able to achieve a much higher sensitivity for detecting $B$. divergens on a given farm than farmers' observations and the existing NDHRS can provide. At all the farms where samples with $1 / 40(+++)$ were detected there were also samples positive at $1 / 160$, indicating that these are real positives. Therefore, by setting a cut-off level of 1:40 (+++) for defining a case of seropositivity for $B$. divergens, antibody testing should result in a specificity of nearly $100 \%$, unless cross-reacting Babesia spp. are occurring and, consequently, the risk of falsely concluding that $B$. divergens occurs on a farm will be small. The related species B. capreoli cause babesiosis in roe deer and red deer [42], and roe deer may also be infected by the newly discovered Babesia sp. EU1 [43]. These parasites cannot be serologically distinguished from $B$. divergens. They cannot give clinical infection in cattle, but there is a possibility that a subclinical infection may cause seroconversion [44], although Schmid et al. [45] found no seropositive cows in an area in which ticks positive for 
these two non-bovine Babesia species were found. It is therefore unlikely that these Babesia species would influence the number of seropositive cows in this study significantly. There are no published studies on these Babesia species in Norway, but a Swedish study suggested that babesiosis caused by $B$. capreoli is very rare in Sweden [46].

An alternative to antibody testing is to test directly for the presence of the pathogen in cattle blood samples. Calder et al. [47] found an approximately $80 \%$ sensitivity for detecting Babesia bovis by PCR in steers, up to 300 days after experimental infection. The method these investigators used required a concentration step involving ultracentrifugation of haemolysed blood. We considered this to be too laborious a method to be useful as a field assay. We did attempt direct PCR to detect $B$. divergens without performing the concentration step in 30 samples from areas where we found the highest incidence of $B$. divergens by IFAT, but none of these samples were found to be $B$. divergens-positive by this method. Cultivation of Babesia in cell culture, which enables detection of Babesia at a level of 10 parasites per $1 \mathrm{ml}$ of blood [48], is another possibility for mapping the distribution of this parasite, but it is not feasible to use this method when sampling is being carried out in scattered locations. For our purposes, therefore, we found that antibody screening was a much more convenient method for assessment of the occurrence of $B$. divergens in a locality than any of the other methods that are available for detecting this pathogen. Gerbilderived antigen is found to be equally specific to $B$. divergens obtained from cattle [49], and could be a cheaper alternative in future studies.

In the communities on the coast of southern Norway where cows are pastured, the animals are confined to the farms on which they are kept. Consequently, testing cows for the presence of $B$. divergens infection should provide results that are specific to a given locality, as opposed to performing serological testing on other hosts of tick-borne pathogens, such as wildlife, dogs or humans. Because $B$. divergence is unlikely to survive for more than a decade in regions where cattle are not pasturing and cattle is the only host for $B$. divergens at the Southern coast of Norway, testing cow sera appears to be an effective method for mapping $B$. divergens over the area of distribution of $I$. ricinus. The same is not the case if using cattle as sentinel animals for serological testing for other tick-borne pathogens, such as $A n a-$ plasma, Borrelia or the TBE virus, that infect a wider range of hosts.

Malandrin et al. [48] found a drop in IFAT antibody titre from 320, 320 and 1280 to 80,80 and 320 respectively in samples from three cows taken 6 and 9 months after acute babesiosis, indicating an antibody duration of more than a year, but much shorter than the cows' lifespan. Sahibi et al. [50] found no significant cumulative effect of cow age on the presence of Babesia-antibodies. This is consistent with a short duration of antibodies in the bloodstream after infection, meaning that detection of antibodies indicates a recent infection, as is illustrated by the seasonal variation of $B$. divergens-antibodies that was found by l'Hostis et al. [38], indicating repeated infections during the season. This implies that the lifetime risk of acquiring bovine babesiosis is higher than the current rate of infection that was determined in the study we present here.

Our IFAT data indicate that there are two areas along the southern Norwegian coast in which bovine babesiosis is highly endemic, consisting of one western area (Lista-Mandal) and one eastern area (Kroger-Risør) (Figure 1, Table 1). This uneven distribution was not reported by Thambs-Lyche in a study carried out along the same part of coastal Norway [29]. For other Babesia species, it has been shown that reduction of the incidence of tick bites can bring the reproduction rate of the parasite below 1, indicating that it could be possible to eradicate the parasite $[41,51]$. Our results indicate that, in the area from Sandaled to Arundel, which is within the distribution area of $I$. ricinus and is an area where cattle are pastured in a natural setting, $B$. divergens occurs at very low frequencies or not at all. In fact, the disease associated with this pathogen has virtually disappeared since the $1930 \mathrm{~s}$, when Thambs-Lyche reported babesiosis in this area. This seems promising with regard to the possibility of eradicating this disease. An attempt to eradicate the disease would require the implementation of control measures over its entire distribution because wild hosts can spread infected ticks. Cervid animals are the most important hosts for adult ticks [52]. Red deer, roe deer and moose have yearly migratory ranges of 200, 100 and 50-60 kilometres respectively [53], and Cervid animals, therefore, have the potential for transporting large numbers of ticks over long distances. Furthermore, birds can transport ticks across geographical barriers. In a recent study, $7.3 \%$ of northward migratory passerine birds were found to carry one or more ticks [54], so, in a situation where cows are pastured in an area that is free of $B$. divergens, or where there is an unstable population of the pathogen, $B$. divergens could conceivably be introduced by birds.

\section{Conclusions}

At present, bovine babesiosis is a limited animal health problem in Norway. The most obvious possible cause of the decline in incidence since the $1930 \mathrm{~s}$ is changes in the use of pastures. Changes in legislation leading to increased use of wood pasturing may reverse the decline 
in incidence, and we may also see a climate-related increase. An increased incidence of $B$. divergens in cattle could have important economic and animal welfare consequences, and further studies are needed to evaluate whether it would be cost effective to implement preventive measures against the spread of this pathogen. Antibody testing of pastured cows is a simple way of mapping the distribution of the pathogen.

\section{Acknowledgements}

Thanks to Ph. D. student Lise Heyer, Department of Biology, University of Oslo, Norway, for help with statistical analyses.

\section{Author details}

'Department of Biology, University of Oslo, P.O. Box 1050 Blindern, N-0316 Oslo, Norway. ${ }^{2}$ Institute for Health and Society, Faculty of Medicine, University of Oslo, Norway. ${ }^{3}$ Department of Virology, Immunobiology and Parasitology. National Veterinary Institute, Uppsala, Sweden. ${ }^{4}$ Department of Basic Sciences and Aquatic Medicine, Norwegian School of Veterinary Science, Norway. ${ }^{5}$ Department of Cattle Health Services, TINE Norwegian Dairy Association, Norway.

\section{Authors' contributions}

$\mathrm{GH}$ prepared the fieldwork, interviewed the farmers, performed all the blood sampling and wrote the main part of the paper. GB, KHR and HPL provided valuable and significant contributions to the writing of the paper. DC headed the laboratory work, and performed all the microscopy of the slides in the immunofluorescence antibody test. Furthermore, he contributed significantly to the writing of the paper. ACW contributed with data from the Norwegian Dairy Herd Recording System, and also contributed significantly to the writing of the paper. All authors read and approved the final manuscript

\section{Competing interests}

The authors declare that they have no competing interests.

Received: 12 April 2010 Accepted: 6 October 2010 Published: 6 October 2010

\section{References}

1. Acha P, Szyfres B: Parasitoses. Zoonoses and Communicable Diseases Common to Man and Animals Washington DC: Pan American Health Organization (PAHO), Third 2003, 2.

2. Bock R, Jackson L, De Vos A, Jorgensen W: Babesiosis of cattle. Parasitology 2005, 129:247-269.

3. Zintl A, Mulcahy G, Skerrett HE, Taylor SM, Gray JS: Babesia divergens, a bovine blood parasite of veterinary and zoonotic importance. Clin Microbiol Rev 2003, 16:622-636.

4. Purnell RE: Bovine babesiosis in the European Community. Veterinary Science Communications 1977, 1:289-296.

5. Christensson DA: Inverse age resistance to experimental Babesia divergens infection in cattle. Acta Vet Scand 1989, 30:453-464.

6. Zintl A, Gray JS, Skerrett HE, Mulcahy G: Possible mechanisms underlying age-related resistance to bovine babesiosis. Parasite Immunol 2005, 27:115-120.

7. Donnelly J, Joyner LP, Crossman PJ: Incidence of Babesia divergens infection in a herd of cattle as measured by the indirect immunofluorescent antibody test. Res Vet Sci 1972, 13:511-514.

8. Taylor SM: Assessment of prevalence of clinical babesiosis in cattle in Northern Ireland. Vet Rec 1983, 112:247-250

9. Gorenflot A, Moubri K, Precigout E, Carcy B, Schetters TP: Human babesiosis. Annals of Tropical Medicine and Parasitology 1998, 92:489-501.

10. García-Sanmartín J, Aurtenetxe O, Barral M, Marco I, Lavin S, García-Pérez AL, Hurtado A: Molecular detection and characterization of piroplasms infecting cervids and chamois in Northern Spain. Parasitology 2007, 134:391-398.
11. Darja Duh, Miroslav Petrovec, Andrej Bidovec, Avsic-Zupanc T: Cervids as Babesiae Hosts, Slovenia. Emerg Infect Dis 2005, 11:1121-1123.

12. Jaenson TGT, Talleklint L, Lundqvist L, Olsen B, Chirico J, Mejlon H: Geographical distribution, host associations, and vector roles of ticks (Acari: Ixodidae, Argasidae) in Sweden. J Med Entomol 1994, 31:240-256.

13. Donnelly J, Peirce MA: Experiments on the transmission of Babesia divergens to cattle by the tick Ixodes ricinus. Int I Parasitol 1975, 5:363-367.

14. Bonnet S, Jouglin M, Malandrin L, Becker C, Agoulon A, L'Hostis M, Chauvin A: Transstadial and transovarial persistence of Babesia divergens DNA in Ixodes ricinus ticks fed on infected blood in a new skin-feeding technique. Parasitology 2007, 134:197-207.

15. Randolph SE: Ticks are not insects: Consequences of contrasting vector biology for transmission potential. Parasitol Today 1998, 14:186-192.

16. Joyner LP, Davies SFM: Acquired resistance to Babesia divergens in experimental calves. J Protozool 1967, 14:260.

17. Chauvin A, Moreau E, Bonnet S, Plantard O, Malandrin L: Babesia and its hosts: adaptation to long-lasting interactions as a way to achieve efficient transmission. Vet Res 2009, 40:37.

18. Thambs-Lyche H: Ixodes ricinus og piroplasmosen I Norge. Norsk Veterinæertidsskrift 1943, 60:337-366.

19. Solbu H: Disease recording in Norwegian dairy cattle. I. Disease incidences and non-genetic effects on mastitis, ketosis and milk fever. $Z$ Tierzuecht Zuechtungsbiol 1983, 100:139-157.

20. Husdyr på utmarksbeite 2006. [http://www.ssb.no/emner/10/04/ jordbruk_miljo/tabeller4.2/beitedyr_2006_00.html].

21. Talet på storfe og sau per 1. januar, etter fylke 2010. [http://www.ssb.no/ emner/10/04/10/jordhus/tab-2010-04-08-02.html].

22. Gray J, Harte L, Talty P: Decline of Bovine Babesiosis in Ireland. Irish Veterinary Journal 1996, 49:157-159.

23. Wesenberg GR: In Terapianbefaling: Antiparasittærbehandling av produksjonsdyr. Edited by: legemiddelverk S. Statens legemiddelverk: Oslo; 2001:

24. Lindgren $E$, Talleklint $L$, Polfeldt T: Impact of climatic change on the northern latitude limit and population density of the diseasetransmitting European tick Ixodes ricinus. Environ Health Perspect 2000, 108:119-123.

25. Forskrift om hold av storfe. [http://www.lovdata.no/cgi-wift/ldles?doc=/sf/ sf/sf-20040422-0665.html\#10].

26. Lundsett AL: Flåtten Ixodes ricinus som sykdomsvektor i Sør-Norge Telemark University College 2004.

27. Radzijevskaja J, Paulauskas A, Rosef O: Prevalence of Anaplasma phagocytophilum and Babesia divergens in Ixodes ricinus ticks from Lithuania and Norway. Int J Med Microbiol 2008, 298:218-221.

28. Ross JPJ, Lohr KF: Serological diagnosis of Babesia bigemina infection in cattle by the indirect fluorescent antibody test. Res Vet Sci 1968, 9:557.

29. Thambs-Lyche H: Ixodes ricinus og piroplasmosen i Norge. Norsk Veterinæertidsskrift 1943, 60:401-441.

30. The Norwegian Surveillance System for Communicable Diseases (MSIS). [http://www.msis.no/].

31. Christensson DA: Improvement of the teflonized slide used in the immunoflourescent antibody technique. Acta Vet Scand 1986, 27:296-297.

32. Christensson DA: A modified IF-test to demonstrate IgM antibodies to Babesia divergens of cattle. Acta Vet Scand 1987, 28:361-371.

33. Christensson DA, Moren T: Seroresponse (IgG) after vaccination and natural infection of cattle with Babesia divergens. Acta Vet Scand 1987, 28:393-402.

34. Clopper CJ, Pearson ES: The use of confidence or fiducial limits illustrated in the case of the binomial. Biometrika 1934, 26:404-413.

35. Christensson DA: Babesia of cattle and sheep in Sweden. 1989.

36. Mørk T, Sviland S: Flåttbårne sykdommer hos storfe: Babesiose. 2009

37. Christensson D, Enfors E: An outbreak of babesiosis (B. divergens) in a dairy herd comprising different age groups of cattle. Acta Vet Scand 1987, 28:125-126.

38. I'Hostis M, Chauvin A, Valentin A, Precigout E, Gorenflot A: Survey of Babesia divergens antibody kinetics in cattle in western France. Vet Res 1997, 28:481-488.

39. Taylor SM, Kenny J, Strain A: The distribution of Babesia divergens infection within the cattle population of Northern Ireland. British Veterinary Journal 1982, 138:384-392. 
40. Gray JS, Harte LN: An estimation of the prevalence and economic importance of clinical bovine babesiosis in the Republic of Ireland. Irish Veterinary Journal 1985, 39:75-78.

41. Mahoney DF, Ross DR: Epizootiological factors in the control of bovine babesiosis. Aust Vet J 1972, 48:292

42. Gray JS, Murphy TM, Taylor SM, Blewett DA, Harrington R: Comparative morphological and cross transmission studies with bovine and deer babesias in Ireland. Preventive Veterinary Medicine, 1990, 9:185-193.

43. Bonnet $\mathrm{S}$, Jouglin M, L'Hostis M, Chauvin A: Babesia sp. EU1 from Roe Deer and Transmission within Ixodes ricinus. Emerging Infectious Diseases 2007, 13.

44. Adam KMG, Blewett DA: The isolation and characterization of a Babesia from red deer (Cervus elaphus). Parasitology 1976, 73:1-11.

45. Schmid N, Deplazes P, Hoby S, Ryser-Degiorgis M-P, Edelhofer R, Mathis A: Babesia divergens-like organisms from free-ranging chamois (Rupicapra r. rupicapra) and roe deer (Capreolus c. capreolus) are distinct from B. divergens of cattle origin - An epidemiological and molecular genetic investigation. Vet Parasitol 2008, 154:14-20.

46. Aguirre AA, Bröjer C, Mörner T: Descriptive epidemiology of roe dee mortality in Sweden. Journal of Wildlife Diseases 1999, 35:753-762.

47. Calder JAM, Reddy GR, Chieves L, Courtney CH, Littell R, Livengood JR, Norval RAl, Smith C, Dame JB: Monitoring Babesia bovis infections in cattle by using PCR-based tests. J Clin Microbiol 1996, 34:2748-2755.

48. Malandrin L, L'Hostis M, Chauvin A: Isolation of Babesia divergens from carrier cattle blood using in vitro culture. Vet Res 2004, 35:131-139.

49. Gray JS, Kaye B: Studies on the use of gerbil-derived Babesia divergens antigen for diagnosis of bovine babesiosis. Vet Parasitol 1991, 39:215-224.

50. Sahibi H, Rhalem A, Berrag B, Goff WL: Bovine babesiosis. Seroprevalence and ticks associated with cattle from two different regions of Morocco. Ann N Y Acad Sci 1998, 849:213-8.

51. Bowman D: Successful and currently ongoing parasite eradication programs. Vet Parasitol 2006, 139:293-307.

52. Ostfeld R, Canham C, Oggenfuss K, Winchcombe R, Keesing F: Climate, deer, rodents, and acorns as determinants of variation in Lyme-disease risk. PLOS Biol 2006, 4:1058-1068.

53. Reimers E: Hjortedyr. In Norges dyr. Edited by: Semb-Johansson A. Oslo: Cappelens forlag AS; 1990:

54. Hasle G, Bjune G, Edvardsen E, Jakobsen C, Linnehol B, Røer J, Mehl R, Røed K, Pedersen J, Leinas H: Transport of ticks by migratory passerine birds to Norway. J Parasitol 2009, 95:1342-1351.

doi:10.1186/1751-0147-52-55

Cite this article as: Hasle et al:: Detection of Babesia divergens in southern Norway by using an immunofluorescence antibody test in cow sera. Acta Veterinaria Scandinavica 2010 52:55.

\section{Submit your next manuscript to BioMed Central and take full advantage of:}

- Convenient online submission

- Thorough peer review

- No space constraints or color figure charges

- Immediate publication on acceptance

- Inclusion in PubMed, CAS, Scopus and Google Scholar

- Research which is freely available for redistribution 